\title{
Properties of an Alginate Gel Bead Containing a Chitosan-Drug Salt
}

\author{
Yoshifumi Murata, * Daijirou Hirai, Kyoko Kofuji, Etsuko Miyamoto, and Susumu Kawashima \\ Faculty of Pharmaceutical Science, Hokuriku University; Ho-3, Kanagawa-machi, Kanazawa 920-1181, Japan. \\ Received September 30, 2003; accepted December 19, 2003
}

\begin{abstract}
A calcium-induced alginate gel bead (Alg-CS) containing chitosan (CS) and 2-(4-chlorophenoxy)-2-methylpropionic acid (CMP) was prepared. We then investigated (a) CMP release from Alg-CS, and (b) uptake of bile acid into the Alg-CS, within the gastrointestinal tract. Dried Alg-CS gradually swelled in taurocholate solution, while releasing CMP and taking up bile acid. The amount of bile acid taken up into the Alg-CS increased incrementally according to the degree of deacetylation of CS. Furthermore, the molecular weight of CS also affected the properties of the Alg-CS. An approximately linear relationship was observed between CMP release and bile acid uptake of Alg-CS.
\end{abstract}

Key words chitosan; drug release; bile acid adsorption; alginate gel bead

Chitosan (CS) is a polysaccharide with polycationic properties, and CS salts is one of anion-exchange resin. ${ }^{1)}$ Although CS is not absorbed in the human gastrointestinal tract, it is an attractive material for drug delivery due to its hypocholesterolemic effect. ${ }^{2)}$ Several physicochemical mechanisms have been proposed to explain this effect of CS, including a decrease in viscosity, as well as a decrease in cholesterol absorption by preventing emulsification of neutral lipids, among others. ${ }^{3-6)}$ In a previous report, we observed that CS adsorbs bile acids due to ion-exchange between CS salts and bile acids. ${ }^{7)}$ For example, upon oral administration of a CS-orotic acid salt to rats, orotic acid, which is a watersoluble vitamin involved in lipid metabolism, was released from the salt, resulting in decreased serum cholesterol. ${ }^{8,9)}$

Alginate, an anionic polysaccharide, forms a cured gel matrix in the presence of calcium (Alg-Ca). Alg-Ca is able to incorporate drug, or another polysaccharide such as CS, within its gel matrix. ${ }^{10,11)}$ Thus, it is thought that Alg-Ca might allow for controlled drug release and uptake of substances dissolved in aqueous medium. In the present study, we prepared Alg-Ca containing CS and 2-(4-chlorophenoxy)-2-methylpropionic acid (CMP). CMP is an acidic compound whose derivatives have been used to treat hyperlipidemia. We then investigated (a) CMP release, and (b) uptake of bile acids, within the gastrointestinal tract, in vitro.

\section{MATERIALS AND METHODS}

Chemicals CMP was purchased from Aldrich Chem. Co. (Milwaukee) and bezafibrate was from ICN Biomedicals Inc. (Ohio). One type of CS (CSF, fine powder, degree of deacetylation (DA): 75-85\%) and Chitin (fine powder) were obtained from Kimitsu Chem. Ind. (Tokyo), while four types of CS (CS10, CS100, CS500, CS1000) were obtained from Wako Pure Chemical Industries, Ltd. (Osaka). Four additional types of CS (7B: DA 70\%, 8B: DA $80 \%$, 9B: DA $90 \%$, 10B: DA 100\%) were obtained from Katokichi Co. Ltd. (Kagawa). Sodium alginate, taurocholate (TCA), and glycocholate (GCA) were purchased from Nacalai Tesque (Kyoto). All other chemicals were of reagent grade.

Determination of CS Molecular Weight by Gel Permeation Chromatography The chromatography system included an LC-10AS pump (Shimadzu), a packed column (Shodex SB-806M HQ, $300 \mathrm{~mm} \times 8.0 \mathrm{~mm}$ i.d.), and a refrac- tive index detector (Shimadzu RID-10A). Chromatography was performed at ambient temperature using $0.1 \mathrm{~m}$ acetate buffer $(\mathrm{pH} 4.5)$ as the eluent at a flow rate of $0.5 \mathrm{ml} / \mathrm{min}$. Eight species of pullulans (Showa Denko K.K., Special chemical Division, MW 5900-790000) were used to prepare the standard curve of molecular weight.

Preparation of Alg-CS Alg-CS was prepared as follows: $0.5 \mathrm{~g}$ of CS were dispersed in $9.5 \mathrm{~g}$ of $1(\mathrm{w} / \mathrm{w}) \%$ sodium alginate solution with agitation. This solution $(2 \mathrm{~g})$ was dropped into $10 \mathrm{ml}$ of $0.1 \mathrm{M} \mathrm{CaCl}_{2}$, and left to stand at room temperature for $1 \mathrm{~h}$. Next, the spherical hydrogel beads ( $4.2 \pm 0.2 \mathrm{~mm}$ in diameter, $n=27)$ were transferred to $50 \mathrm{ml}$ of distilled demineralized water containing $0.01-0.2 \mathrm{~g} \mathrm{CMP}$, which was autoclaved at $121^{\circ} \mathrm{C}$ for $15 \mathrm{~min}$ (or left to stand at room temperature for $1 \mathrm{~d})$. Dried Alg-CS beads $(2.7 \pm 0.2 \mathrm{~mm}$ in diameter) were prepared as follows: Alg-CS (hydrogel) beads were taken out and dried at $35^{\circ} \mathrm{C}$ for $8 \mathrm{~h}$ on a dish, followed by vacuum treatment in a desiccator in the presence of $\mathrm{P}_{2} \mathrm{O}_{5}$.

Test of CMP Release and Bile Acid Uptake Fifteen milliliters of a $2 \mathrm{~mm}$ bile acid solution or demineralized water were placed in an L-shaped glass tube and maintained at $37^{\circ} \mathrm{C}$. An amount of dried Alg-CS beads corresponding to $2 \mathrm{~g}$ of hydrogel was added to the solution and shaken 67 times per min. A $0.2 \mathrm{ml}$ aliquot of each solution was removed periodically (after 15, 30, 45, 60, 90 and $120 \mathrm{~min}$ ) to determine the amount of CMP and bile acid by HPLC analysis at the same time. The HPLC system was comprised of an LC6A pump (Shimadzu, Kyoto), a packed column (Mightysil RP-18 GP, $150 \mathrm{~mm} \times 4.6 \mathrm{~mm}$, Kanto Chem. Co., Tokyo), and a SPD-6A UV detector (Shimadzu). HPLC was conducted at ambient temperature, using an eluent containing methanol, $30 \mathrm{~mm}$ phosphate buffer ( $\mathrm{pH} 3.4)$, and acetonitrile $(6: 3: 1)$, at a flow rate of $0.6 \mathrm{ml} / \mathrm{min}$, and the detector wavelength was set at $230 \mathrm{~nm}$. The amount of bile acid taken up into the AlgCS was calculated based on the amount of bile acid added and that remaining at sampling time. All tests were performed in triplicate.

Drug Solubility Drug solubility was measured by adding the sample to distilled demineralized water and shaking at $37^{\circ} \mathrm{C}$ using a shaker (MONOSIN-IIA, TAITEC, Saitama). The solution was then removed and subjected to rapid filtration, followed by dilution. The amount of drug in solution was determined by the HPLC method described 


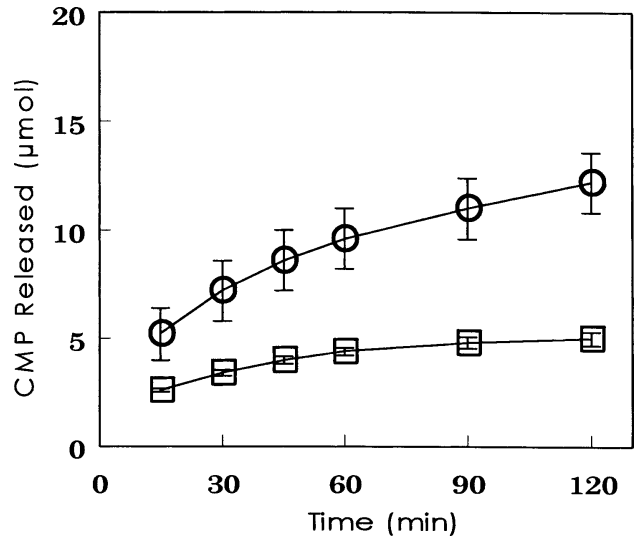

Fig. 1. CMP Release from Alg-CS(F) in Distilled Water ( $\square)$ or $2 \mathrm{~mm}$ TCA (O)

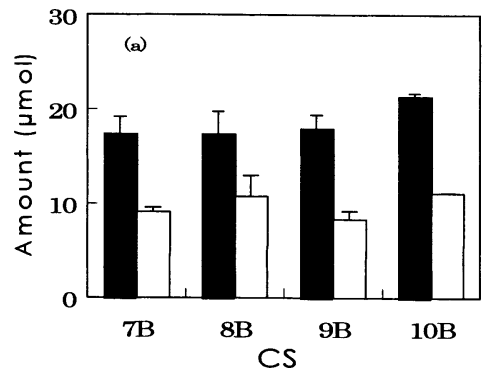

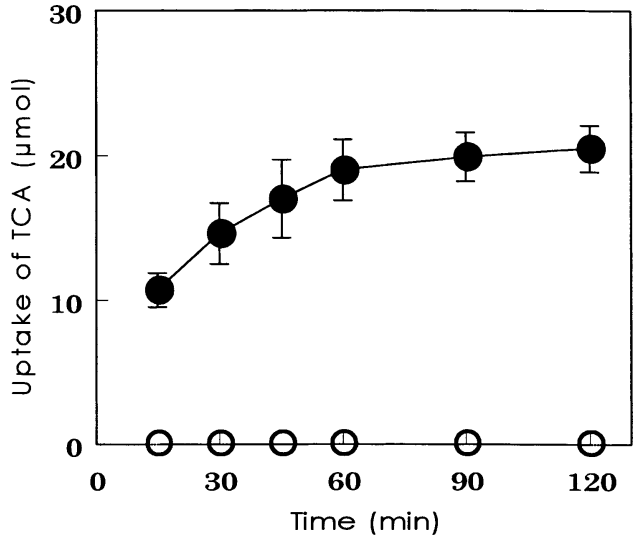

Fig. 2. Uptake of TCA into Alg-CS(F)

-, Autoclaved in $0.1(\mathrm{mg} / \mathrm{ml}) \mathrm{CMP}$; , autoclaved in distilled water.

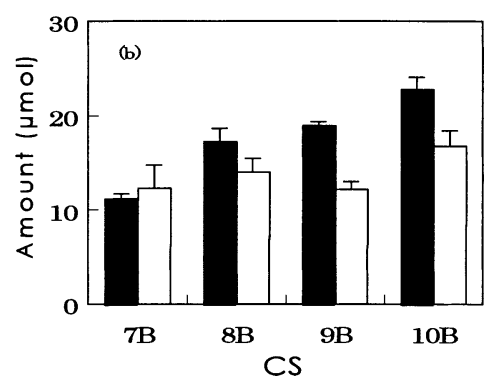

Fig. 3. Effect of Deacetylation of CS on CMP Release (Open Column) and Uptake of TCA (Closed Column) at $2 \mathrm{~h}$

(a) Autoclaved, (b) stood at room temperature.

above.

\section{RESULTS AND DISCUSSION}

Dried Alg-CS containing 5\% CSF, designated Alg-CS(F), swelled in demineralized water to a diameter of $3.3 \pm 0.2 \mathrm{~mm}$ $(n=27)$ after $2 \mathrm{~h}$. This led to rapid release of $5.0 \pm 0.3 \mu \mathrm{mol}$ of CMP from the gel matrix at $2 \mathrm{~h}$, as shown in Fig. 1. However, when Alg-CS(F) was incubated in $2 \mathrm{~mm}$ TCA solution, a much larger quantity of CMP was released $(12.2 \pm 1.4 \mu \mathrm{mol}$ at $2 \mathrm{~h}$ ). In addition, $1.8 \pm 0.4 \mu \mathrm{mol}$ of CMP was released from Alg-Ca (CS-free) in TCA solution, while 6.6 $\pm 0.2 \mu \mathrm{mol}$ was released from Alg-Ca containing 5\% chitin. These results indicate that the amino groups of $\mathrm{CS}$ determine how much $\mathrm{CMP}$ is released from $\mathrm{Alg}-\mathrm{CS}$.

Alg-CS gradually adsorbed bile acid dissolved in the test solution. Fig. 2 shows the uptake of TCA by Alg-CS(F). After $1 \mathrm{~h}, 19.0 \pm 2.1 \mu \mathrm{mol}$ of TCA was taken up into Alg$\mathrm{CS}(\mathrm{F})$, theoretically containing $0.1 \mathrm{~g}$ of CS. Uptake of glycocholate was also observed, with uptake of $18.0 \pm 0.7 \mu \mathrm{mol}$ at $1 \mathrm{~h}$ and $21.5 \pm 1.1 \mu \mathrm{mol}$ at $2 \mathrm{~h}$. No uptake of TCA was observed into Alg-CS autoclaved with water, which did not contain CMP. Moreover, Alg-CS(F) treated with bezafibrate instead of CMP took up extremely little bile acid. The watersolubility of bezafibrate $(38 \mu \mathrm{g} / \mathrm{ml})$, compared with that of CMP $(850 \mu \mathrm{g} / \mathrm{ml})$, might be too low for adequate CS salt formation.

Figure 3a shows the effect of DA of CS on CMP release and TCA uptake from Alg-CS. The most uptake of TCA was observed with Alg-CS(10B). However, differences in TCA uptake were not observed among Alg-CS(7B), Alg-CS(8B)
Table 1. Effect of CS Molecular Weight (MW) on Release of CMP and Uptake of TCA at $2 \mathrm{~h}($ mean \pm S.D., $n=3)$

\begin{tabular}{ccccr}
\hline \hline & \multicolumn{4}{c}{ Species } \\
\cline { 2 - 5 } MW & CS10 & CS100 & CS500 & \multicolumn{1}{c}{ CS1000 } \\
(Dispersion) & 67000 & 314000 & 514000 & 1036000 \\
& $(4.0)$ & $(3.7)$ & $(2.9)$ & $(2.9)$ \\
\hline Dried gel & & & & \\
CMP $(\mu \mathrm{mol})$ & $10.6 \pm 0.8$ & $12.2 \pm 1.4$ & $6.8 \pm 0.4$ & $9.0 \pm 0.4$ \\
TCA $(\mu \mathrm{mol})$ & $19.7 \pm 1.4$ & $23.4 \pm 2.2$ & $15.6 \pm 1.2$ & $17.2 \pm 1.3$ \\
Hydrogel & & & & \\
CMP $(\mu \mathrm{mol})$ & $13.8 \pm 0.2$ & $13.4 \pm 0.0$ & $7.2 \pm 0.6$ & $8.2 \pm 0.2$ \\
TCA $(\mu \mathrm{mol})$ & $20.6 \pm 0.2$ & $26.2 \pm 0.3$ & $16.2 \pm 1.7$ & $18.6 \pm 1.1$ \\
\hline
\end{tabular}

and $\mathrm{Alg}-\mathrm{CS}(9 \mathrm{~B})$. When Alg-CS was prepared at room temperature, TCA uptake into Alg-CS(7B) was limited, as shown in Fig. 3b. This result shows that CS salt formation within an alginate gel matrix might be accelerated by autoclaving AlgCS.

The molecular weight of CS affected the properties of the Alg-CS gel, as shown in Table 1. Lesser amounts of CMP were released from gel beads containing high-molecular weight CS species, such as CS500 and CS1000, than from those made with low-molecular weight CS species. Moreover, uptake of bile acid into Alg-CS accelerated according to increment of the amount of CMP released. This tendency has also been observed with hydrogel beads. Uptake of TCA into hydrogel beads is similar to that of dried gel beads, therefore, uptake might be governed by CS characteristics, rather than the water content of the gel matrix surrounded CS and CMP. 


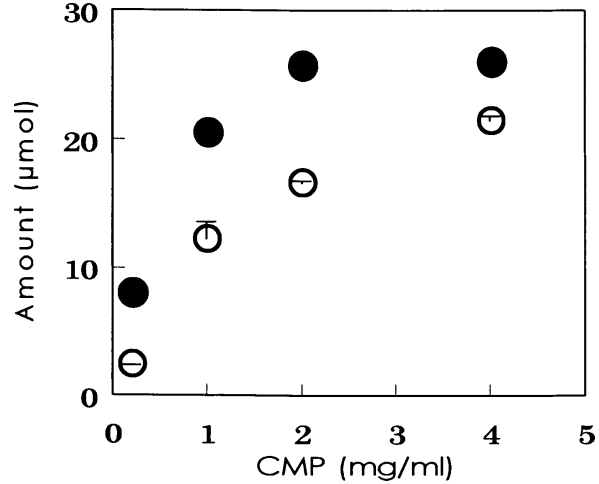

Fig. 4. Effect of CMP Concentration on CMP Release from Alg-CS (F) and the Uptake of TCA at $2 \mathrm{~h}$

$\bigcirc$, CMP release; $\boldsymbol{\bullet}$, uptake of TCA.

Greater drug release was observed as more CMP was incorporated into the Alg-CS matrix, as shown in Fig. 4. TCA uptake was also observed to depend on CMP concentration and the amount taken into Alg-CS prepared with $2 \mathrm{mg} / \mathrm{ml}$ CMP went up to $85 \%$ of bile acid dissolved in the medium. Furthermore, an approximately linear relationship between the amount of CMP released at $2 \mathrm{~h}$ and the amount of TCA taken up by each Alg-CS bead was observed, as shown in Fig. 5.

In this study, we prepared Alg-CS containing CMP, which was capable of drug release and bile acid uptake via an ionexchange reaction. Both of these functions reduce cholesterol and/or triacylglycerol levels in plasma. Therefore, Alg-CS might be a useful agent by which to treat hyperlipidemia, which is primarily a lifestyle-related disease. ${ }^{12)}$

Acknowledgments This work was supported in part by a grant from Hokuriku University.

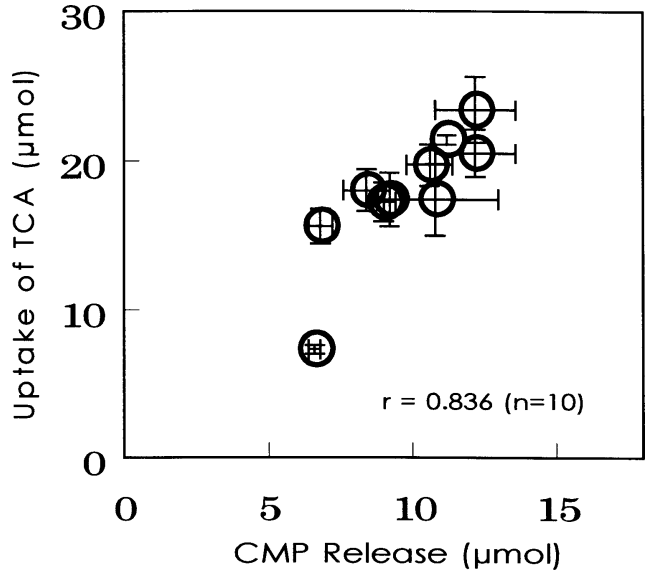

Fig. 5. Relationship between CMP Release and Uptake of TCA

\section{REFERENCES}

1) Shu X. Z., Zhu K. J., Int. J. Pharmaceut., 21, 217-225 (2002).

2) Shields K. M., Smock N., McQueen C. E., Bryant P. J., Am. J. Health Syst. Pharm., 60, 1312-1316 (2003).

3) Gallaher C. M., Munion J., Hesslink R., Jr., Wise J., Gallaher D. D., J. Nutrition, 130, 2753-2759 (2000).

4) Ylitalo R., Lehtinen S., Wuolijoki E., Ylitalo P., Lehtimaki T., Arzneimittelforschung, 52, 1-7 (2002).

5) Barroso A. J., Contreras F., Bagchi D., Preuss H. G., J. Med., 33, $209-225$ (2002).

6) Hejazi R., Amiji M., Pharm. Dev. Technol., 8, 253-262 (2003).

7) Murata Y., Toniwa S., Miyamoto E., Kawashima S., Int. J. Pharmaceut., 176, 265-268 (1999).

8) Aoyama Y., Morifuji M., J. Nutr. Sci. Vitaminol., 48, 40-46 (2002).

9) Murata Y., Kojima N., Kawashima S., Biol. Pharm. Bull., 26, 687690 (2003).

10) Iskakov R. M., Kikuchi A., Okano T., J. Control. Release, 80, 57-68 (2002).

11) Murata Y., Tsumoto K., Kofuji K., Kawashima S., Chem. Pharm. Bull., 51, 218-220 (2003).

12) Homma Y., Kobayashi T., Yamaguchi H., Ozawa H., Sakane H., Nakamura H., Atherosclerosis, 21, 241—248 (1997). 